\title{
Geometry effects on the interaction of two equal-sized drops in simple shear flow at finite Reynolds numbers
}

\author{
S. Mortazavi \& M. Bayareh \\ Department of Mechanical Engineering, \\ Isfahan University of Technology, Iran
}

\begin{abstract}
The effect of geometry on the interaction of two equal-sized drops in shear flow is presented. The full Navier-Stokes equations are solved by a finite difference/front tracking method. The interaction of drops was studied at finite Reynolds numbers for viscosity ratio $(\lambda)$ of one. The distance between drop centres along the velocity gradient direction (z) was measured as a function of time. The interaction of two drops contains approach, collision, and separation. Based on experimental data, we simulated different geometries by changing the offset and size of drops. It was found that $\Delta z$ increases after collision and reaches to a new steady-state value after separation. The values of $\Delta z$, during three stages of interaction, increases with the increasing initial offset. To investigate the drop shape evolution, we calculated the deformation and the orientation angle formed by the drop major axis and horizontal direction. The deformation of the drops is maximum when the drops are pressed against each other and minimum when they are drawn a part. Our results show that the time of approaching of drops at low initial offset is greater than the other ones, but the maximum deformation is the same for equal drop sizes. The deformation decreases with the decreasing size of drops. As the initial offset increases, the drops rotate more quickly and the available contact time for film drainage decreases. We found that the trajectories of drops in the approaching stage are different owing to the different initial offsets. However, after the drops come into contact, it can be seen that they follow the same trajectories, similar to experimental results.
\end{abstract}

Keywords: two-phase flow, front tracking, Reynolds number, Weber number, capillary number, offset, drainage time. 


\section{Introduction}

Immiscible polymer blending plays a fundamental role in determining material properties of industrial interest. It is important to understand and control the size and size distribution of the dispersed drops because the properties of the blend depend on them. The final size distribution is determined by a balance between flow-induced break up and coalescence. While break-up involves a single drop and is not very affected by the presence of neighbouring drops in a blend (Leowenberg and Hinch [1], coalescence is the result of many-particle interaction process.

The majority of numerical simulations are based on the interaction of two deformable drops in a shear flow, the drainage of the thin film between two colliding drops and the problems of coalescence of two deformable drops. Wang et al. [2] computed the coalescence of two undeformed spherical drops. Leowenberg and Hinch [3] presented the numerical simulations of the interaction between deformable drops based on boundary integral calculations. They showed that if capillary number is much smaller than one, the tendency for coalescence is greatest when drops are pressed against each other by the shear flow. Viscosity ratio effects on film drainage between interacting drops were studied by Bazhlekov et al. [4]. Cristini et al. [5] simulated the drop break-up and coalescence by an adaptive mesh algorithm. Effects of inertia on the rheology of a dilute emulsion of drops in shear flow are investigated by Zhao [6] using direct numerical simulation. The drop shape and flow are computed by solving the Navier-Stokes equations in two phases using front tracking method.

On the other hand, most of experimental works are based on blending studies that analyses the drop size distribution of a blend or a concentrated emulsion. The collision of two equal-sized drops immersed in an immiscible liquid phase undergoing a shear flow in a parallel apparatus was investigated by Guido and Simeone [7] over a range of capillary numbers. Trajectories of a pair drops and their deformations were presented. The coalescence efficiency of two drops in a simple shear flow was also investigated by Mousa et al. [8]. The effect of viscosity ratio on the flow-induced coalescence of two equal-sized drops with clean interfaces was investigated by Yoon et al. [9]. Their studies showed that when the viscosity ratio is greater than $\mathrm{O}(0.1)$, the critical capillary number decreases with increasing offset only for the smallest offset. Zhao [10] investigated the drop break up in dilute Newtonian emulsions in simple shear flow by using high-speed microscopy over a wide range of viscosity ratio, focusing on high capillary number. He showed the final drop size distribution intimately links to the drop break up mechanism, which depends on viscosity ratio and capillary number.

In this article, we present numerical simulation data describing the motion of a pair of drops under simple shear flow at finite Reynolds numbers. We consider the special case of drops with the same viscosity as the continues-phase fluid. Formulation and numerical method are described in $\S 2$, results are presented in $\S 3$, and concluding remarks are given in $\S 4$. 


\section{Formulation and numerical method}

\subsection{Formulation}

The governing equations for the motion of unsteady, viscous, incompressible, immiscible two-fluid systems are the Navier-Stokes equations in conservative form:

$$
\frac{\partial \rho u}{\partial t}+\nabla \cdot \rho u u=-\nabla P+\nabla \cdot \mu\left(\nabla u+\nabla u^{T}\right)+\sigma \int \kappa n \delta^{\beta}(x-X) d_{s} .
$$

Here $\mathrm{u}$ is the fluid velocity, $\mathrm{p}$ is the pressure, $\rho$ is the fluid density, $\mu$ is the fluid viscosity, $\sigma$ is the surface tension coefficient, $g$ is the acceleration due to gravity. $\delta^{\beta}$ is a two- or three-dimensional delta function (for $\beta=2$ and $\beta=3$ ) respectively. $\kappa$ is the curvature for two-dimensional flows and twice the mean curvature for three-dimensional flows. $\mathrm{n}$ is a unit vector normal to the drop surface pointing outside of the drop. $\mathrm{x}$ is the position in Eulerian coordinate and $\mathrm{X}$ is the position of front in Lagrangian coordinate.

Both of immiscible fluids are taken to be incompressible, so the divergence of velocity field is zero:

$$
\nabla \cdot u=0 .
$$

Equations of state for the density and the viscosity are:

$$
\frac{D \rho}{D t}=0, \quad \frac{D \mu}{D t}=0 .
$$

Continuity of stresses at the fluid boundary shows that the normal stresses are balanced by surface tension. The force due to surface tension is

$$
\Delta F=\sigma k n \text {. }
$$

Three governing non-dimensional numbers of the flow are the Reynolds number (bulk and particle Reynolds numbers), the Weber number and the capillary number. Only two of these non-dimensional numbers are independent (one Reynolds number and the Weber or capillary number):

$$
\operatorname{Re}_{b}=\frac{\rho_{0} G H^{2}}{\mu_{0}}, \quad \operatorname{Re}_{P}=\frac{\rho_{0} G R^{2}}{\mu_{0}}, \quad W e=\frac{\rho_{0} G^{2} R^{3}}{\sigma}, \quad C a=\frac{\mu_{0} G R}{\sigma} .
$$

Here $\rho_{0}$ is the density of ambient fluid, $\mu_{0}$ is the viscosity of the ambient fluid, $\mathrm{R}$ is the initial radius of the drop, $\mathrm{H}$ is the width of the channel and $\mathrm{G}$ is the shear rate. The shear rate is

$$
G=\frac{u_{t}-u_{b}}{H}
$$

where $u_{t}$ and $u_{b}$ are the velocity of top and bottom walls, respectively.

It is usual to define a scalar measure of the drop deformation (the Taylor deformation) by:

$$
D=\frac{l-b}{l+b} .
$$

where $1, \mathrm{~b}$ are the major and minor semi-axes of the drop (defined by the largest and smallest distances of the surface from the centre).

In addition, the collision or film drainage time is the time between the points where the centre-to-centre distance is equal to one undeformed drop diameter to the instant of coalescence. 


\subsection{Numerical method}

Various methods have been used to simulate the two-phase flows. These methods include the Marker-And-Cell (MAC) method, the Volume-Of-Fluid (VOF) method, and the level set method. In general, the interface representation can be explicit (moving mesh) or implicit (fixed mesh) or a combination of both. The front-tracking method is combination of fixed and moving mesh method. Although an interface grid tracks the interface, the flow is solved on a fixed grid. The interface conditions are satisfied by smoothing the interface discontinuities and interpolating interface forces from the interface grid to the fixed grid. In this method, the governing equations are solved separately for each fluid. Front capturing has two difficulties. The first is a sharp boundary between the fluids and the second is accurate computation of surface tension. Different attempts have been made in overcoming these problems.

For the simulations presented here, the method developed by Unverdi and Tryggvason [11] is used. They simulated the motion of buoyant bubbles in a periodic domain. Eqns (1), (2), and (3) are solved in a rectangular threedimensional domain with a finite difference method. The spatial differentiation is calculated by a second order finite difference scheme on a staggered Eulerian grid. We use an explicit second-order time integration method.

Combining the incompressibility condition and momentum equations results in a non-separable elliptic equation for the pressure. Due to the similarity in density between the drop and the ambient fluid, a quick poisson solver solves the pressure equation. The force due to surface tension on each element of front is

$$
\delta F_{\sigma}=\int_{\Delta S} \sigma \kappa n d_{S} .
$$

In three-dimensional flow, the average surface curvature is

$$
\kappa n=(n \times \nabla) \times n .
$$

Then, the force on each element surface is

$$
\delta F_{\sigma}=\sigma \int_{\sigma A} \kappa n d_{A}=\sigma \int_{\sigma A}(n \times \nabla) \times n d_{A}=\sigma \oint_{S} t \times n d_{S} .
$$

The integration is over the boundary of each element representing the front. $t$ and $n$ are the tangent and the normal vector to each element, respectively.

\section{Results}

The reference system to describe the results is shown in fig. 1. According to experiments of Yoon et al. [9], initial offset is defined as the shortest distance from the centre of the drop to the inflow axis $(\Delta)$ divided by drop radius $R$. The centre-to-centre distance between drops is $4 \mathrm{R}$ as shown in fig. 1. The coordinate axes are oriented as follows: the $\mathrm{x}$-axis is parallel to flow direction, the $\mathrm{y}$-axis is parallel to the vorticity direction, and the z-axis is parallel to the velocity gradient. The relative trajectory of the two drops will be expressed in terms of the differences $\Delta z=z_{2}-z_{1}$ and $\Delta x=x_{2}-x_{1}$, where $x_{i}$ and $z_{i}$ are the centre-of- 
mass coordinates of the ith drop. The difference $\Delta y$ between the y-coordinates of the two drop centres is zero.

In all plots, $\Delta \mathrm{x}$ and $\Delta \mathrm{z}$ will be made dimensionless by the radius $\mathrm{R}$ of the undeformed drops as the characteristic length. We will compare our results with experimental results of Guido and Simeone [7] and Yoon et al. [9] and numerical results of Loewenberg and Hinch [3]. Sequences (1-4) show the interactions between two drops in simple shear flow (fig. 2). Initially, each drop has the steady shape under same flow conditions.

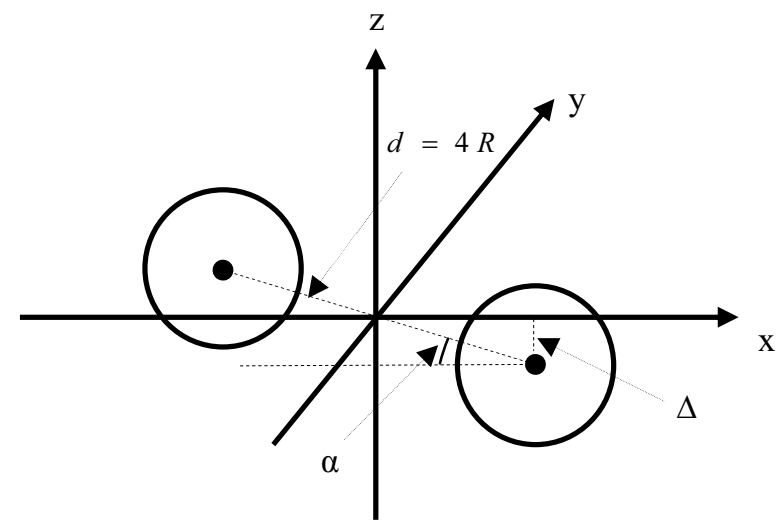

Figure 1: $\quad$ Schematic of the relative trajectory between a pair of deformable interacting drops in shear flow (Offset $=\Delta / \mathrm{R})$.
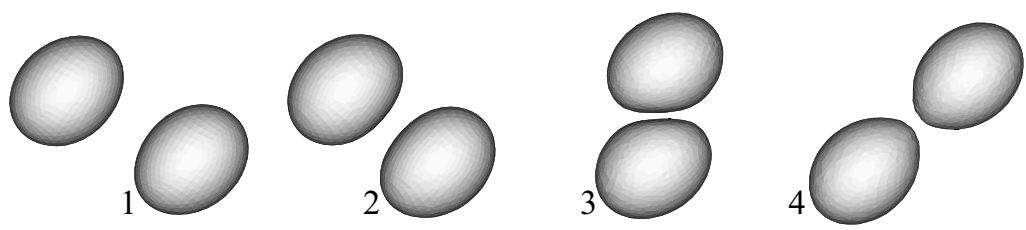

Figure 2: $\quad$ Sequences (1-4) showing the interaction between two drops in simple shear flow with $\mathrm{Ca}=0.13$, Offset $=0.512, \lambda=1$.

In fig. $3, \Delta \mathrm{z}$ is plotted as a function of $\Delta \mathrm{x}$ during approach, collision and separation between two drops. The data correspond to sequence depicted in fig. 2. It can be seen that $\Delta z$ starts increasing after the drops come into apparent contact $(\Delta x \sim-2 R)$, reaches a maximum value, and, after separation, reaches a new steady-state value.

The final value of $\Delta z$ (which is 1.4 for offset $=0.2,1.52$ for offset $=0.512$, and 1.72 for offset $=0.8$ ) is greater than the value before collision. In the other words, if the drops were made to collide again by reversing the flow direction, $\Delta \mathrm{z}$ increased further. So, the effect was irreversible, and repeated collisions lead to increasing values of $\Delta z$ until drop interaction became negligible (Guido and 
Simeone [7]). This is also an agreement with the numerical simulations of Loewenberg and Hinch [3]. Experimental results of Guido and Simeone [7] was based on $\lambda=1.4$ and numerical results of Loewenberg and Hinch [3] were presented for viscosity ratio of one.

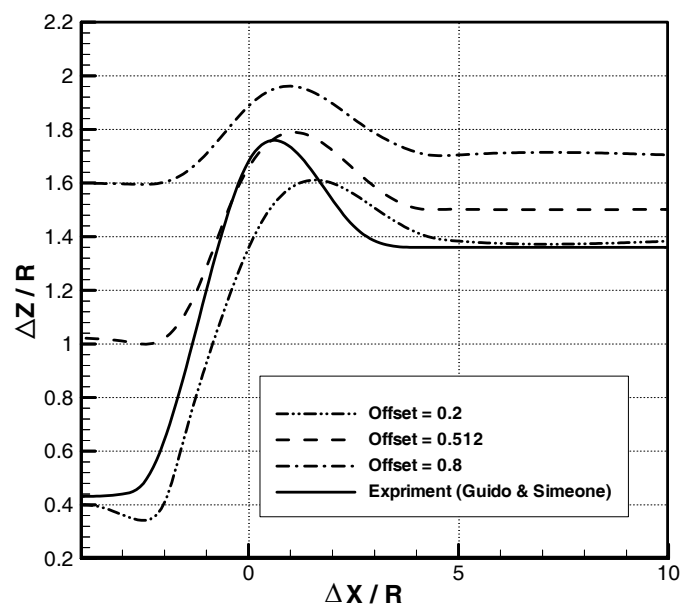

Figure 3: Cross-flow separation (velocity gradient direction) versus $\Delta \mathrm{x} / \mathrm{R}$ between interacting drops with $\mathrm{Ca}=0.13, \lambda=1$ (present work) and $\lambda=1.4$ (experiment), and different offsets.

The deformation parameter is shown in fig. 4 as a function of dimensionless time with viscosity ratio of one and $\mathrm{Ca}=0.3$. Based on experimental observation of Guido and Simeone [7], deformation of two drops is the same. Deformation slightly increases, and then reaches a maximum, a minimum, a second maximum, and eventually reaches a steady state value from before the collision. Numerical simulations of Loewenberg and Hinch [3] show no difference before and after collision at low-Reynolds numbers. Comparison between the results shows that the time of approaching of drops at low initial offset is greater (for current simulations).

Following Allan and Mason [12] and Guido and Simeone [7], the minimum value of deformation is lower than the steady-state value before (or after) collision. This can be explained as the result of two processes: (i) relaxation of drop shape once they leave the compressional axis and (ii) action of the surrounding fluid on the drops.

At fixed initial offsets, as the size of drops increases, the deformation increases as shown in fig. 5. We see that the approach-collision-separation times of drops are different owing to the different initial sizes. The film drainage time increases with increasing size of drops. The dimensionless drainage time is 5.21 for $\mathrm{D} / \mathrm{H}$ $=0.3$ and 5.86 for $\mathrm{D} / \mathrm{H}=0.36$.

Fig. 6 shows the trajectories of the drops for different initial offsets with the same capillary number for a viscosity ratio of 1 . As the initial offset increases, 
the drops rotate more quickly and the available time for film drainage decreases as shown in fig. 6. Therefore, we should expect that the critical capillary number for coalescence will decrease with increasing offset. This was found in studies of Yang et al. [13], where the viscosity ratio was 0.096 .

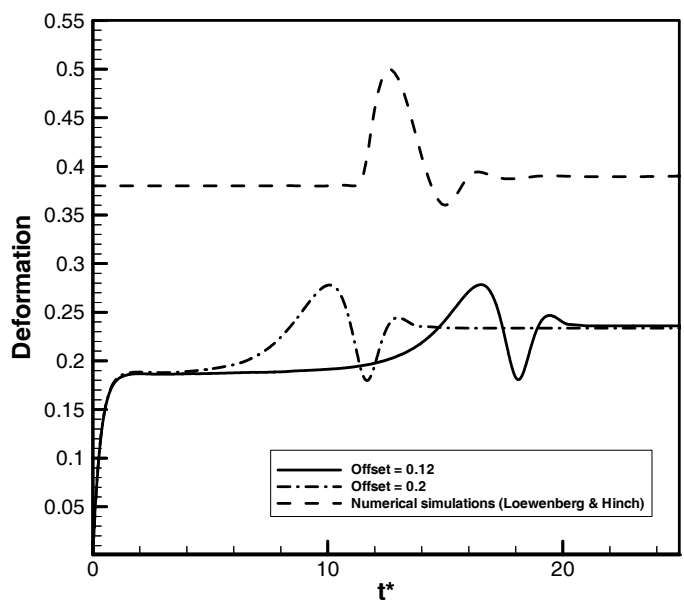

Figure 4: The deformation parameter as a function of dimensionless time between interacting drops with $\mathrm{Ca}=0.3, \lambda=1$ and different offsets.

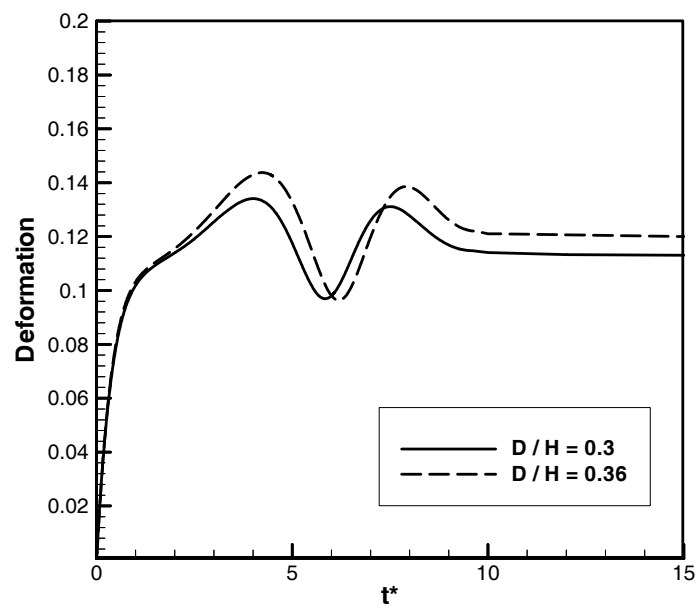

Figure 5: The deformation parameter as a function of dimensionless time between interacting drops with $\mathrm{Ca}=0.075, \lambda=1$ and different size of drops.

In fig. 7 we see that the approaching parts of the trajectories are different from each other owing to different initial offsets. However, after the drops come into 
contact, it can be seen that they follow the same trajectories of separation distance versus orientation angle $(\alpha)$ formed by the drop major axis and horizontal direction. This is an agreement with the experimental results of Yoon et al. (2005). They said that they have no explanation for this result.

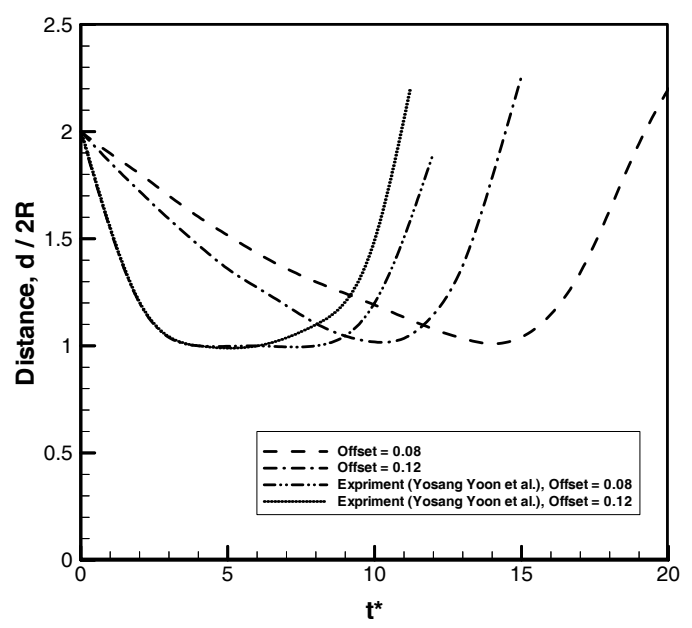

Figure 6: Trajectories of drops for different initial offsets, separation distance versus dimensionless time, with $\mathrm{Ca}=0.00481, \lambda=1$ (present work), and $\lambda=1.2$ (experiment).

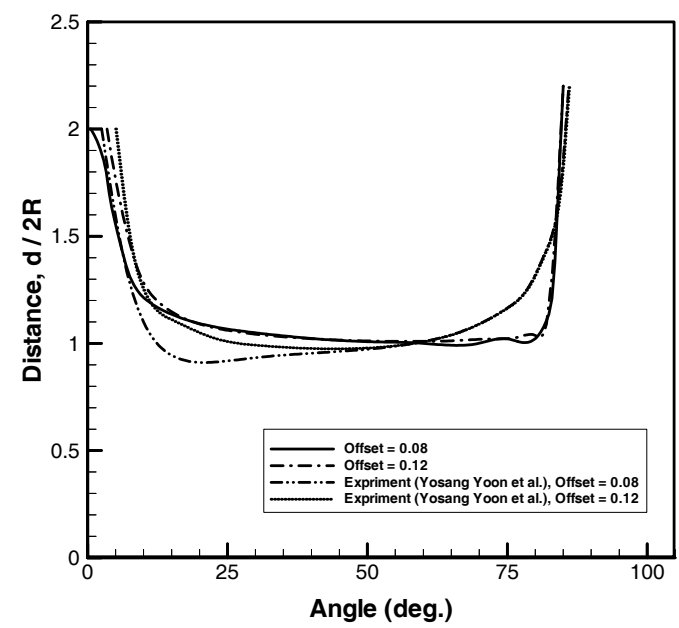

Figure 7: Trajectories of drops for different initial offsets, separation distance versus orientation angle $(\alpha)$, with $\mathrm{Ca}=0.00481, \lambda=1$ (present work), and $\lambda=1.2$ (experiment). 


\section{Conclusion remarks}

The effects of geometry include initial offset and size of drops on the interaction of two equal-sized drops in simple shear flow has been presented using finite difference/front tracking method. Simulations were studied at finite Reynolds numbers for viscosity ratio of one.

The deformation, relative trajectories, and film drainage time were examined by changing the initial offset and size of drops. We changed offset and size of drops, Based on experimental data. It was found that $\Delta \mathrm{z}$ increases after collision and reaches to a new steady-state value after separation. The values of $\Delta z$, during the interaction, increases with the increasing initial offset.

Our results showed that the time of approaching of drops at low initial offset is greater than the other ones, but the maximum of deformation is the same for equal drop sizes. The deformation decreases with the decreasing size of drops. As the initial offset increases, that time for film drainage decreases. Also, the approaching parts of the trajectories are different from each other owing to the different initial offsets. However, after the drops come into contact, it can be seen that they follow the same trajectories of separation distance versus orientation angle formed by the drop major axis and horizontal direction, similar to experimental results.

\section{References}

[1] Loewenberg, M. \& Hinch, E.J., Numerical simulation of a concentrated emulsion in shear flow. J. Fluid Mech., 321, pp. 395-419, 1996.

[2] Wang, H., Zinchenko, A.Z. \& Davis, R.H., The collision rate of small drops in linear flow-fields. J. Fluid Mech., 265, pp. 161-188, 1994.

[3] Loewenberg, M. \& Hinch, E.J., Collision of two deformable drops in shear flow. J. Fluid Mech., 338, pp. 299-315, 1997.

[4] Bazhiekov, I.B., Chesters, A.K. \& Van De Vosse, F.N., The effect of the dispersed to continuous-phase viscosity ratio on film drainage between interacting drops. Intl J. Multiphase Flow, 26, pp. 445-466, 2000.

[5] Cristini, V., Blawzdziewicz, J. \& Loewenberg, M., An adaptive mesh algorithm for evolving surface: simulation of drop break-up and coalescence. J. Comput. Phys. 168, pp. 445-463, 2001.

[6] Zhao, X., Effects of inertia on the rheology of a dilute emulsion of drops in shear flow. J. Rheology, 49, pp. 1377-1394, 2005.

[7] Guido, S. \& Simeone, M., Binary collision of drops in simple shear flow by computer-assisted video optical microscopy. J. Fluid Mech., 357, pp. 1-20, 1998.

[8] Moua, H., Agterof, W. \& Mellema, J., Experimental investigation of the orthogenetic coalescence efficiency of droplets in simple shear flow. $J$. Colloid Interface Sci., 240, pp. 340-348, 2001.

[9] Yoon, Y., Borrell, M., Park, C.C. \& Leal L.G., Viscosity ratio effects on the coalescence of two equal-sized drops in a two-dimensional linear flow. J. Fluid Mech., 525, pp. 355-379, 2005. 
[10] Zhao, X., Drop break up in dilute Newtonian emulsions in simple shear flow: new drop break up mechanism. J. Rheology, 51, pp. 367-192, 2007.

[11] Unverdi, S.O. \& Tryggvason, G., Computations of multi-fluid flows. Phys. Fluids, D60, pp. 70-83, 1992.

[12] Allan, R.S. \& Mason, S.G., Particle motion in sheared suspensions. XIV. Coalescence of liquid drops in electric and shear fields. J. Colloid Interface Sci., 17, pp. 383-408, 1962.

[13] Yang, H., Park, C.C, Hu, Y.T. \& Leal, L.G., The coalescence of two equalsized drops in a two-dimensional linear flow. Phys. Fluids, 13, pp. 10871106, 2001. 